\title{
Efeito da Sequência de Mistura nas Propriedades de Blendas PA6/ABS Compatibilizadas com o Copolímero SMA
}

\author{
Amanda D. de Oliveira, Nelson M. Larocca, Luiz A. Pessan \\ Departamento de Engenharia de Materiais, UFSCar
}

\begin{abstract}
Resumo: Blendas de poliamida 6 (PA6) com acrilonitrila-butadieno-estireno (ABS) foram preparadas em uma extrusora de rosca dupla, utilizando-se o copolímero estireno-anidrido maleico (SMA), com 7\% de anidrido maleico, como compatibilizante. O efeito de três sequências de mistura na microestrutura e propriedades das blendas foi estudado. A morfologia e as propriedades mecânicas dos materiais foram caracterizadas por microscopia eletrônica de transmissão (MET) e testes de resistência ao impacto e módulo de elasticidade, respectivamente. Os resultados mostraram que a morfologia e propriedades mecânicas das blendas ternárias foram bastante diferentes e dependem da sequência de mistura. A blenda preparada pela mistura simultânea de todos os componentes, em uma única extrusão, apresentou melhor tenacidade. Por outro lado, quando o ABS e o SMA foram misturados juntos em uma primeira extrusão, antes da incorporação da PA6 em uma segunda extrusão, o valor da resistência ao impacto encontrado foi menor que o da matriz PA6 e da blenda sem compatibilizante PA6/ABS. Evidências de reações químicas entre o compatibilizante SMA e a matriz PA6 também foram investigadas através de reometria de torque.
\end{abstract}

Palavras-chave: Blendas, poliamida 6, tenacidade, sequência de mistura.

\section{Effect from the Blending Sequence on the Properties of PA6/ABS Blends Compatibilized with SMA Copolymer}

Abstract: Blends of polyamide 6 (PA6) with acrylonitrile-butadiene-estyrene (ABS) were prepared in a twin-screw extruder, using the styrene-maleic anhydride (SMA) copolymer containing 7\% of maleic anhydride as compatibilizer. The effects from three blending sequences on the microstructure and properties of the blends were investigated. The morphology and mechanical properties of the materials were characterized by transmission electron microscopy and tensile and impact tests. The results showed that the morphology and mechanical properties of ternary blends depend on the sequence of blend preparation. The blend prepared using the mixture of all components in a single-pass extrusion showed the more significant improvement in the material toughness. However, when the ABS and SMA were melt mixed together in a first extrusion step prior to incorporating PA6 in a second extrusion, the impact strength is lower than that of the blend without compatibilizer PA6/ABS and PA6 matrix. Evidence of chemical reactions between the compatibilizer SMA and PA6 was also obtained using a torque rheometer.

Keywords: Blends, polyamide 6, toughness, blend sequence.

\section{Introdução}

As poliamidas pertencem a uma classe de polímeros atraente para aplicações em engenharia devido à combinação de propriedades como: baixa viscosidade no estado fundido, boa resistência ao impacto sem entalhe e excelente resistência química. Por outro lado, as poliamidas são altamente higroscópicas e sensíveis ao entalhe, isto é, são dúcteis quando não entalhados, mas fraturam de maneira frágil quando entalhados, devido a sua baixa resistência à propagação da trinca. Em adição, poliamidas tendem a ser frágeis em temperaturas subambientes e carregamento sob condições severas. Essas deficiências podem ser melhoradas pela mistura destes polímeros com outros plásticos como poli(óxido de fenileno) (PPO), poli(acrilonitrila-butadieno-estireno) (ABS), poliolefinas, entre outros ${ }^{[1-3]}$. O ABS consiste de uma fase elastomérica dispersa numa matriz termoplástica constituída de estireno e acrilonitrila (SAN). A incorporação do ABS pode melhorar a resistência ao impacto sob entalhe das poliamidas, pois sua fase elastomérica, à base de polibutadieno (PB), apresenta um enorme potencial para aumentar a tenacidade da mistura final, bem como, diminuir a elevada absorção de umidade destes materiais ${ }^{[3,4]}$. Entretanto, como essa mistura é imiscível e incompatível, faz necessário o uso de compatibilizantes capazes de reagir com os grupos terminais da PA6 e que sejam miscíveis com a fase SAN do $\mathrm{ABS}^{[3,5-8]}$.
O grupo anidrido maleico (MA) pode reagir com o grupo terminal amina da PA6. Logo, os polímeros contendo o grupo MA podem efetivamente serem usados no processo de compatibilização reativa da poliamida 6 . O copolímero estireno-anidrido maleico (SMA) pode ser usado como um compatibilizante em blendas com o terpolímero ABS, uma vez que o SMA pode ser misturado em nível molecular com a matriz SAN do ABS quando as quantidades de MA do SMA e acrilonitrila (AN) do ABS não são muito diferentes ${ }^{[9-11]}$. Eles geralmente serão imiscíveis quando essa quantidade for superior a $5 \%{ }^{[6]}$.

Os vários componentes de tais sistemas de blendas tenacificadas na presença de um compatibilizante reativo, podem ser combinados utilizando diferentes sequências de mistura. A literatura mostra que a ordem de mistura dos componentes da blenda apresenta grande influência no resultados de resistência ao impacto, embora não apresente influência significativa nos resultados de comportamento mecânico sob tração ${ }^{[12-14]}$. Vários fatores podem contribuir para esta alteração da tenacidade: a sequência de mistura pode influenciar o curso que as reações químicas ocorrem ou a localização do copolímero em bloco ou enxertado formado, podendo afetar a morfologia e outros fatores que governam a tenacidade ${ }^{[14]}$. O objetivo principal deste trabalho é estudar o efeito da ordem de mistura dos componentes da

Autor para correspondência: Amanda D. de Oliveira, Departamento de Engenharia de Materiais, Universidade Federal de São Carlos,

Rodovia Washington Luis, Km 235, Caixa Postal 676, CEP: 13565-905, São Carlos, SP, Brasil. E-mail: amandaoliveira82@gmail.com 
blenda PA6/ABS/SMA na morfologia e propriedades mecânicas finais destes sistemas. Será mostrado que as sequências de mistura utilizadas afetam significativamente a morfologia e as propriedades mecânicas destas blendas.

\section{Experimental}

\section{Materiais}

A Poliamida 6 (PA6) com índice de fluidez de $29.8 \mathrm{~cm}^{3} / 10 \mathrm{~min}$ $\left(235{ }^{\circ} \mathrm{C} / 2.16 \mathrm{Kg}\right)$, de especificação B300 foi fornecida pela Polyform. O ABS (acrilonitrila-butadieno-estireno), designado comercialmente como Cycolac* Resin EX58, foi fornecido pela SABIC. Apresenta 52,8\% de polibutadieno, $12,4 \%$ de acrilonitrila e $34,6 \%$ de estireno. O copolímero estireno-anidrido maleico (SMA), com 7\% de anidrido maleico (MA) e índice de fluidez 1,9 g/10 min $\left(230{ }^{\circ} \mathrm{C} / 2.16 \mathrm{Kg}\right)$, foi fornecido pela Sigma-Aldrich e utilizado como compatibilizante. Tanto a PA6 como o ABS e o SMA foram fornecidos na forma de grânulos.

\section{Preparação das blendas}

Antes da preparação das misturas a PA6, o ABS e o SMA foram moídos na forma de um pó fino, em moinho criogênico e em seguida submetidos à secagem em estufa sob vácuo, a $80{ }^{\circ} \mathrm{C}$, durante 24 horas. As blendas foram preparadas em uma extrusora de rosca dupla, B\&P Process Equipment Systems, modelo MP19 (L/D $=25, D=19 \mathrm{~mm}$ ), com perfil de temperatura de: 200, 220, $220,220,230{ }^{\circ} \mathrm{C}$ e com rotação da rosca de $160 \mathrm{rpm}$. Os materiais extrudados foram granulados após saída da matriz, secados e moldados por injeção em uma injetora Arburg 270 V, 30 ton., com diâmetro de $25 \mathrm{~mm}$ e volume máximo de injeção $54 \mathrm{~cm}^{3}$. Os corpos de prova foram confeccionados para os ensaios de resistência mecânica à tração, ao impacto Izod e temperatura de deflexão térmica (HDT), de acordo com as normas ASTM D638-02a, ASTM D256-06 e ASTM D 648, respectivamente. A moldagem por injeção foi feita com uma pressão de injeção de 900 bar. O perfil de temperatura utilizado foi $230,240,240,240,245^{\circ} \mathrm{C}$ e a temperatura do molde de $50{ }^{\circ} \mathrm{C}$.

Para estudar o efeito do copolímero SMA na compatibilização da blenda PA6/ABS e na dispersão das partículas de ABS, bem como na morfologia e propriedades finais da mesma, três sequências de mistura foram preparadas:

1. (PA6/ABS/SMA) - significa que os componentes foram alimentados todos juntos na extrusora;

2. $(P A 6 / S M A)+A B S$ - indica que a PA6 foi primeiramente misturada com o SMA; a esta blenda foi adicionado posteriormente o ABS; e

3. $(A B S / S M A)+P A 6$ - indica que o ABS foi misturado primeiramente com SMA e depois adicionou-se a PA6.

Após cada etapa de pré-mistura, exceto para a primeira onde os componentes foram alimentados todos juntos, o material foi moído em moinho criogênico para novamente ser submetido à etapa de extrusão.

Composições com estas sequências de mistura foram preparadas nas proporções 57,5/37,5/5\% (PA6/ABS/SMA) e foram preparadas também misturas binárias PA6/ABS (60/40\%).

\section{Avaliação da reatividade do copolímero SMA}

A análise da reatividade in situ do compatibilizante SMA com a PA6 e o ABS foi realizada em um Reômetro de Torque Haake, operando-se a uma temperatura de $240^{\circ} \mathrm{C}$ e $60 \mathrm{rpm}$, por 10 minutos.

\section{Caracterização mecânica}

Ensaios de tração foram realizados de acordo com a norma ASTM D638-02a, utilizando um equipamento Instron modelo 5568, com extensômetro. O módulo foi medido a uma velocidade de $5,0 \mathrm{~mm} / \mathrm{min}$. Os dados apresentados são uma média de dez amostras.

A resistência ao impacto foi medida em corpos de prova com entalhe utilizando um equipamento de impacto tipo pêndulo CEAST, de acordo com a ASTM D256-06. Oito amostras foram testadas para cada composição para obtenção de um valor médio.

\section{Caracterização morfológica}

A morfologia das blendas foi analisada pela observação de imagens obtidas em um microscópio eletrônico de transmissão (MET). As amostras analisadas foram retiradas de corpos de prova de impacto Izod. A área das amostras observada foi localizada no meio de seu comprimento, na direção perpendicular ao fluxo de injeção. Em seguida essas amostras foram submetidas ao "trimming" (corte na forma de trapézio) e foram criogenicamente microtomadas, ou seja, cortadas em seções ultrafinas, $20 \mathrm{~nm}$ de espessura, com facas de diamante, por um micrótomo tipo Riechert-Jung Ultracut E, em torno de $-50{ }^{\circ} \mathrm{C}$. Foram utilizadas telas ou "grids" de cobre para coletar as amostras fatiadas que se encontravam imersas em uma solução de DMSO:água (3:2). O tingimento da fase polibutadieno e da fase SAN do ABS foi realizado em duas etapas. Inicialmente, as amostras foram expostas ao vapor de tetróxido de ósmio $\left(\mathrm{OsO}_{4}\right)$ por 15 horas, para tingir a fase borrachosa do ABS. A seguir, as amostras foram expostas ao vapor de tetróxido de rutênio $\left(\mathrm{RuO}_{4}\right)$ por 2 horas para tingir a fase SAN. Após o tingimento, os "grids" com as amostras tingidas foram analisados em microscópio eletrônico de transmissão Philips, modelo CM120, a uma voltagem de aceleração de $120 \mathrm{KV}$.

\section{Temperatura de deflexão térmica}

A temperatura de deflexão térmica (HDT) foi obtida através de corpos de prova de HDT, conforme a norma ASTM D 648, em um equipamento Ceast, modelo HDT 6 VICAT P/N 6921.000, com uma tensão de $1800 \mathrm{kPa}$, taxa de aquecimento de $120^{\circ} \mathrm{C} / \mathrm{h}$ (método A), onde o meio de imersão foi um óleo de silicone. A temperatura foi determinada após a amostra ter defletido $0,25 \mathrm{~mm}$. Uma série de seis amostras foi ensaiada e a temperatura de deflexão média, com seu respectivo desvio-padrão, reportados.

\section{Resultados e Discussão}

\section{Análise da reatividade do copolímero SMA com os componentes da blenda polimérica através de reometria de torque}

Um compatibilizante reativo é um copolímero com grupos funcionais reativos que é miscível com um componente da blenda e reage com o outro componente para formar copolímeros em bloco ou enxertado. As reações químicas envolvidas em blendas de PA6 tenacificada, compatibilizadas com o SMA, envolvem os grupos anidrido maleico do copolímero SMA e os grupos terminais amina da PA6 durante a mistura do fundido. A Figura 1 apresenta esta reação. Para atuar como um eficiente compatibilizante, o copolímero formado in situ PA6-g-MA deve se localizar preferencialmente na interface $^{[1,12,15]}$. 
<smiles>C[C@@H]1C(=O)CC(=O)[C@@H]1C</smiles>

SMA

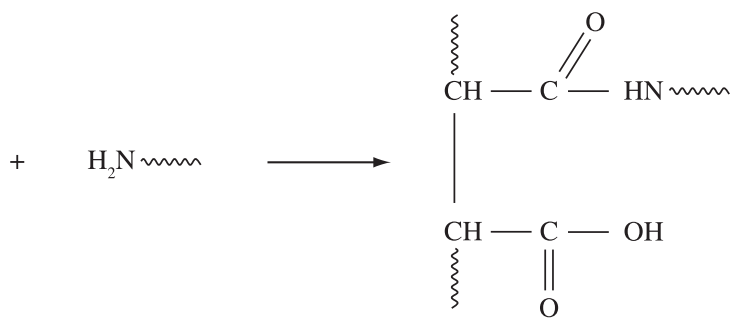

PA6-g-MA

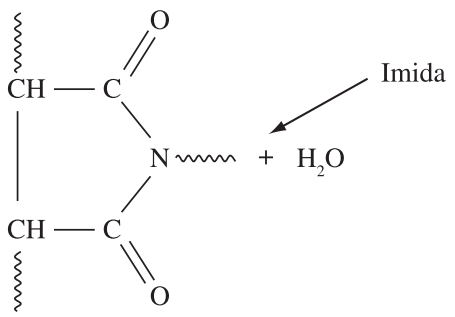

PA6-g-MA

Figura 1. Reação do grupo anidrido do SMA com a amina da PA6, formando uma imida ${ }^{[1]}$.

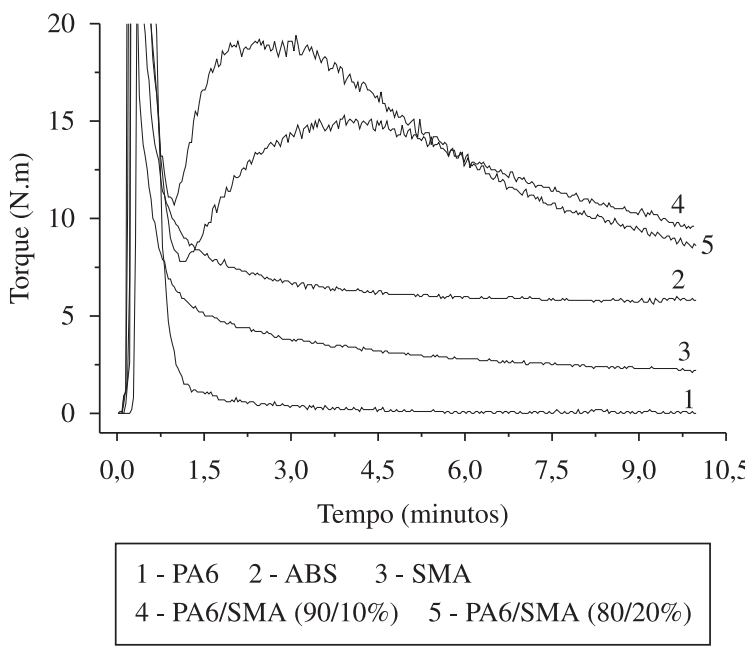

Figura 2. Curvas de torque em função do tempo de processo para as misturas PA6/SMA (90/10 e 80/20\%) medidas a $240{ }^{\circ} \mathrm{C}$ e $60 \mathrm{rpm}$.

A Figura 2 apresenta as curvas de torque em função do tempo para a PA6, ABS, SMA e para o sistema PA6/SMA. Observase que $10 \%$ de copolímero na mistura com a PA6 ocorre um aumento significativo no torque em tempos relativamente curtos, aproximadamente 2 minutos, em seguida, o torque diminui e se mantém praticamente constante. $\mathrm{O}$ aumento da quantidade de SMA na mistura (20\%) é acompanhado por um correspondente aumento no torque. Este fenômeno indica a ocorrência de reações químicas entre o MA do SMA e os grupos finais de cadeia da PA6 e, consequentemente, a formação de moléculas de copolímero PA6-g-SMA, que podem estar localizados na interface entre os componentes. Resultados semelhantes foram encontrados por Choi et al. ${ }^{[13]}$, onde eles avaliaram o torque em função do tempo para misturas PA6/SMA (SMA com 7\% de MA) com teores variados de SMA (1, 5 e 10\%). Os autores observaram que as misturas com maior quantidade de SMA (10\%) apresentaram maior valor do torque em relação às outras, e apresentaram também uma redução mais acentuada do torque com o aumento do tempo de mistura. Eles atribuíram esta redução do torque das misturas com o aumento do tempo, à água formada pela reação de imidização (formação do grupo imida) entre o grupo final amina da PA6 e MA do SMA (Figura 1), a qual pode causar hidrólise do grupo amida da PA6. Esta formação de água parece reduzir a viscosidade da blenda nos estágios subsequentes de mistura e, além disso, ela depende da concentração de SMA.

Um fator que deve ser considerado no nível de reações de enxertia que ocorrem durante a compatibilização reativa é a concentração do grupo reativo. Araújo e colaboradores ${ }^{[16]}$ estudando blendas PA6/ABS e o copolímero MMA-MA (metacrilato de metila- anidrido maleico) como compatibilizante, observaram que quando se tem conteúdos menores de MA, tais como 3 e 5\%, no copolímero são necessárias quantidades maiores de copolímero, por exemplo $20 \%$, para se ter um aumento significativo no torque. Entretanto, com quantidades maiores de MA no copolímero, 10 e 20\%, ocorre exatamente o contrário. Isso pode ser atribuído ao número limitado de grupos funcionais amina presentes na PA que podem reagir com o copolímero. Portanto, não é necessário adicionar grandes quantidades de copolímero para garantir a reação, pois não existem grupos amina suficientes para a reação. Por meio dos resultados apresentados na Figura 2, onde se observa um significativo aumento do torque para as misturas de PA6 com o SMA, acredita-se que 7\% de MA no SMA é suficiente para que ocorra a reação de enxertia.

Blendas de ABS/SMA também foram preparadas com diferentes composições e seus resultados estão apresentados na Figura 3. Pode ser observado que a mistura de ABS com o SMA apresenta um pequeno aumento no torque para altos teores de ABS na blenda ( 80 e $90 \%)$. Este resultado sugere que alguma reação ocorre durante a mistura destes componentes no estado fundido. $\mathrm{Na}$ mistura $90 \%$ de ABS e $10 \%$ de copolímero este aumento no torque já é observado, quando comparado aos valores de torque obtidos para o ABS e o SMA puros, indicando elevação de viscosidade. Aumentando-se a quantidade de SMA para $20 \%$, verifica-se um aumento no torque, indicando que a viscosidade da mistura continua aumentando. Observa-se ainda na Figura 3 que quando o sistema apresenta 40 e $50 \%$ de copolímero, o torque se mantém abaixo daquele das duas misturas anteriormente mencionadas e entre os valores de torque obtidos para os componentes puros. Isto indica que para este sistema ABS/SMA, uma alta quantidade de ABS na blenda é requerida para iniciar o aumento da viscosidade. Como as blendas preparadas neste trabalho apresentam aproximadamente 40\% de ABS e 5\% de SMA, espera-se que estas possíveis reações que ocorrem em sistemas com mais altas quantidades de ABS, não ocorram para as misturas estudadas aqui.

Hale et al. ${ }^{[14]}$, estudando blendas de Poli(tereftalato de butileno) PBT/ABS utilizaram o copolímero MMA-GMA (metacrilato de metila-metacrilato de gricidila), que é miscível com o SAN do ABS, como compatibilizante e observaram um aumento significante do torque para as blendas binárias ABS/MMA-GMA com diferentes composições. A adição de apenas $1 \%$ de ABS nesta blenda já se observa aumento do torque. Eles atribuíram este aumento da viscosidade às possíveis reações que podem ocorrer entre o grupo epóxi do GMA com o ABS. Segundo os autores, o ABS sintetizado pelo processo de polimerização por emulsão na presença de um emulsificante, é composto por ácidos carboxílicos graxos, capazes de reagir com o epóxi. Resultados similares também foram observados por Araújo e colaboradores ${ }^{[16]}$, onde foi utilizada a blenda PA6/ABS compatibilizada com o MMA-GMA. Embora o copolímero SMA utilizado neste trabalho não apresente grupo epóxi, o qual pode reagir com o ABS, a presença desses ácidos no ABS provavelmente 

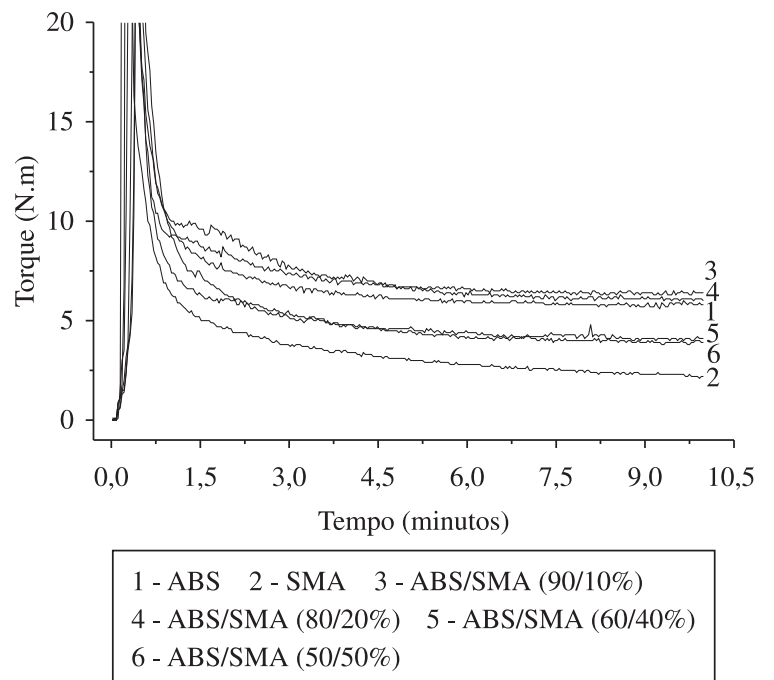

Figura 3. Curvas de torque em função do tempo de processo para as misturas ABS/SMA $(90 / 10,80 / 20,60 / 40$ e $50 / 50 \%)$ medidas a $240{ }^{\circ} \mathrm{C}$ e $60 \mathrm{rpm}$.

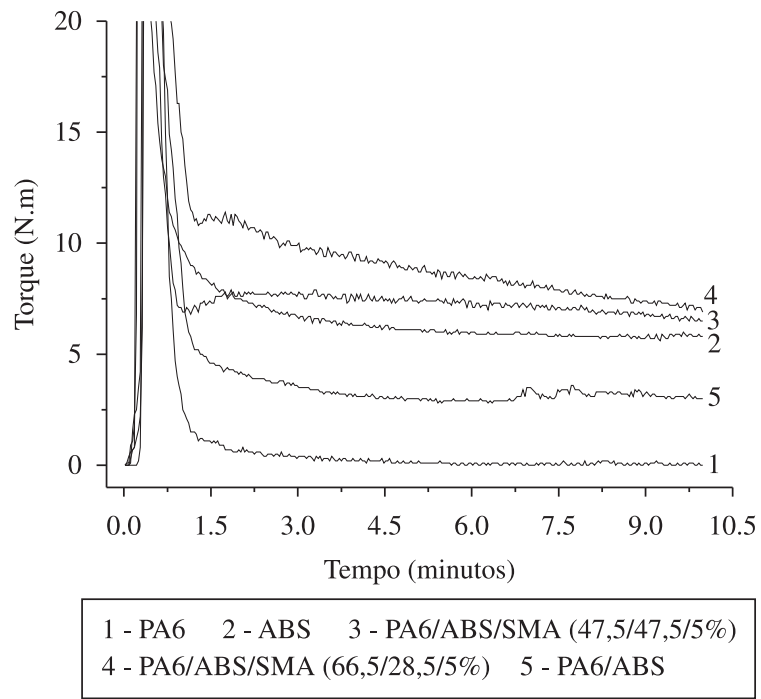

Figura 4. Curvas de torque em função do tempo de processo para a blenda PA6/ABS/SMA medidas a $240{ }^{\circ} \mathrm{C}$ e $60 \mathrm{rpm}$.

seja a causa deste aumento de viscosidade encontrado. Estes resultados precisam ser confirmados em um estudo posterior.

O comportamento das blendas PA6/ABS/SMA também foi estudado, Figura 4, a quantidade de compatibilizante foi mantida fixa (5\%) e variaram-se a razão entre PA6/ABS. Observa-se que há elevação no torque para as blendas compatibilizadas comparadas com a não compatibilizada e os polímeros puros, o que indica que ocorre reação. Essa elevação no torque se torna mais pronunciada com o aumento da quantidade de PA6 na mistura, que eleva o número de grupos funcionais amina presentes para que possam reagir com o MA do SMA.

\section{Caracterização morfológica}

A Figura 5 mostra as micrografias obtidas por MET da blenda binária PA6/ABS e das blendas ternárias com 5\% de SMA. Para uma melhor visualização das fases presentes, a fase polibutadieno foi tingida com tetróxido de ósmio $\left(\mathrm{OsO}_{4}\right)$ e a fase $\mathrm{SAN}$ foi tingida
Tabela 1. Módulo de elasticidade e resistência ao impacto Izod com entalhe das blendas.

\begin{tabular}{ccc}
\hline Material (\%) & $\begin{array}{c}\text { Módulo de } \\
\text { elasticidade (GPa) }\end{array}$ & $\begin{array}{c}\text { Resistência ao } \\
\text { impacto }\left(\mathbf{J}^{-1}\right)\end{array}$ \\
\hline PA6 & $2,7 \pm 0,2$ & $40 \pm 3,3$ \\
ABS & $1,9 \pm 0,05$ & $403 \pm 5,6$ \\
$\begin{array}{c}\text { PA6/ABS } \\
(60 / 40 \%)\end{array}$ & $2,8 \pm 0,03$ & $36 \pm 1,5$ \\
$\begin{array}{c}\text { PA6/ABS/SMA } \\
(57,5 / 37,5 / 5 \%)\end{array}$ & $2,3 \pm 0,1$ & $63,7 \pm 6,4$ \\
$\begin{array}{c}\text { (PA6/SMA) + ABS } \\
(57,5 / 5+37,5 \%)\end{array}$ & $2,5 \pm 0,09$ & $41 \pm 1,8$ \\
$\left(\begin{array}{c}\text { ABS/SMA) + PA6 } \\
(37,5 / 5+57,5 \%)\end{array}\right.$ & $2,3 \pm 0,1$ & $28 \pm 3,4$ \\
\hline
\end{tabular}

tetróxido de rutênio $\left(\mathrm{RuO}_{4}\right)$. Através desta técnica de tingimento é possível observar os domínios esféricos de polibutadieno (partículas escuras) no interior da fase matriz SAN e distinguir a fase PA6 da fase SAN.

Observa-se na Figura 5 que as morfologias apresentadas pelas blendas ternárias, preparadas com diferentes sequências de mistura, são bastante diferentes. Isto indica que a sequência de mistura dos componentes afeta a morfologia da blenda. A blenda PA6/ABS, Figura5a, apresenta domínios de ABS alongados, largos e com uma ampla distribuição de tamanho das partículas de borracha. Quando 5\% do copolímero SMA são introduzidos na blenda, Figura 5b, ocorre uma redução do tamanho desses domínios, embora ainda existam alguns domínios de ABS relativamente alongados. Há tendência à co-continuidade da fase ABS. Por outro lado, a blenda preparada pela sequência (PA6/SMA) + ABS resultou em uma redução mais acentuada do tamanho dos domínios dispersos. Embora essa blenda tenha apresentado melhor dispersão que a blenda preparada pela mistura simultânea de todos os componentes, este sistema não apresentou melhor resultado de resistência ao impacto, uma vez que o valor obtido foi menor que o valor encontrado para a blenda PA6/ABS/SMA (Tabela 1). Provavelmente outros fatores contribuíram para menor tenacidade apresentada por este sistema, como será mostrado mais adiante. Uma causa possível para esta redução dos domínios dispersos observada por esta blenda seria o aumento da viscosidade da fase PA6. Blendas PA6/SMA são sujeitas a reação química durante a mistura do fundido, esta reação resulta em aumento da viscosidade, como mostra os resultados obtidos por meio de reometria de torque (Figura 2). Este efeito altera a razão de viscosidade entre a PA6 e o ABS e resulta em uma mudança na morfologia ${ }^{[12]}$. Para esta sequência de mistura, o SMA foi adicionado à PA6, para depois, em outra etapa de extrusão, ser adicionado o ABS. Este procedimento favorece uma maior reação de enxertia que a mistura simultânea de todos os componentes.

Kudva et al. ${ }^{[5]}$ tem mostrado em seus estudos sobre blendas de PA6/ABS compatibilizadas com o copolímero IA (copolímero acrílico imidizado), que existe um tamanho crítico superior e inferior, além do qual as partículas de borracha não são eficientes para a tenacificação. Segundo os autores, partículas abaixo de um certo limite são incapazes de cavitar, e assim não participam do mecanismo de tenacificação. Talvez esta seja uma possível causa da baixa tenacidade apresentada pela blenda (PA6/SMA) + ABS .

$\mathrm{Na}$ Figura 5d estão apresentadas as micrografias da blenda (ABS/SMA) + PA6 com diferentes ampliações. Os domínios de ABS apresentados por esta sequência de mistura parecem ser maiores e mais alongados que os domínios apresentados pela blenda binária PA6/ABS. Essa alteração na morfologia foi acompanhada por uma redução significativa na resistência ao impacto destas 

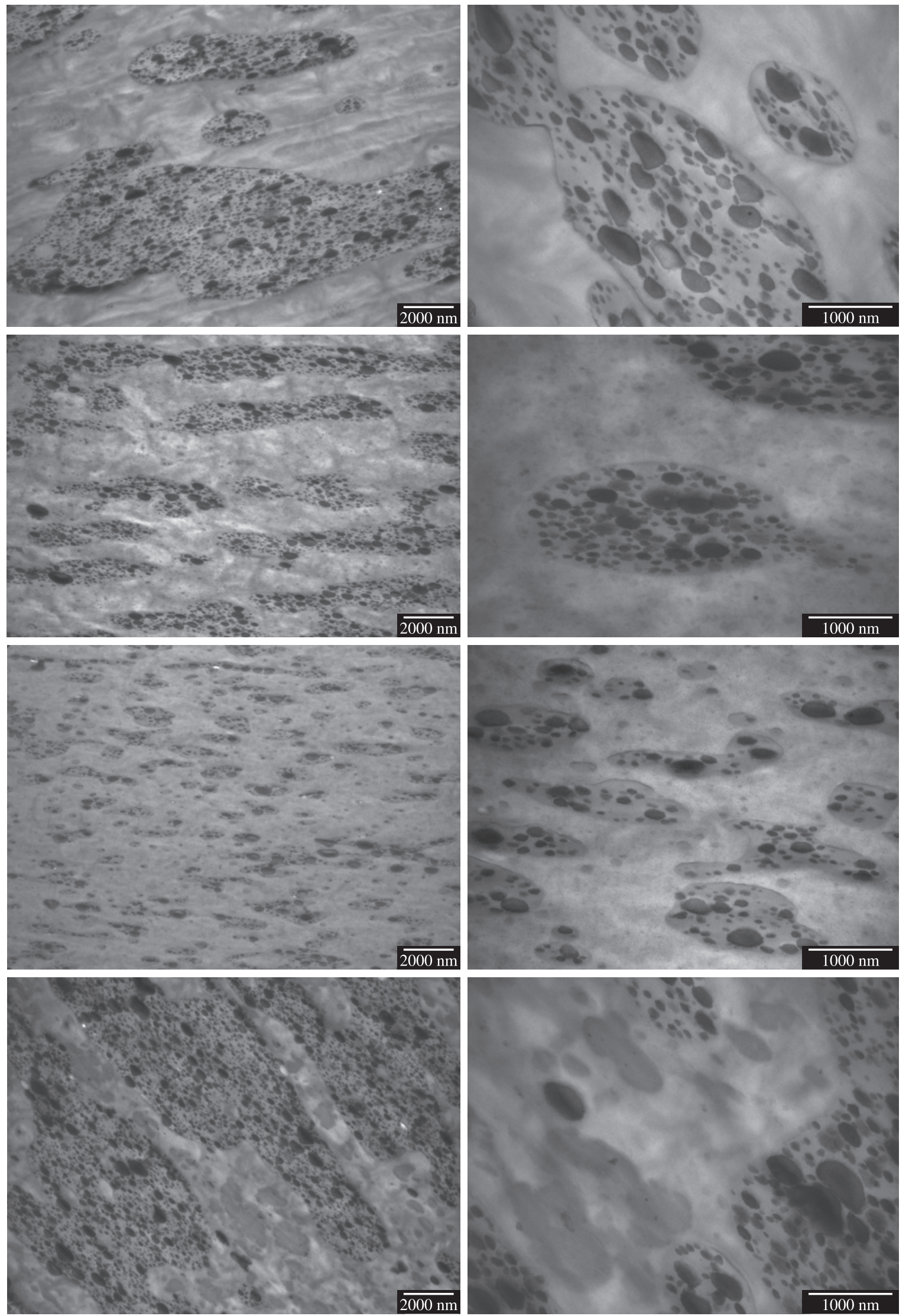

Figura 5. Micrografias obtidas por MET das blendas binárias e ternárias: a) PA6/ABS; b) PA6/ABS/SMA; c) (PA6/SMA) + ABS; e d) (ABS/SMA) + PA6. 
blendas. Observa-se ainda na Figura $5 \mathrm{~d}$ a presença de outra fase dentro da fase matriz PA6. Este efeito também já foi observado na literatura por outros autores ${ }^{[11]}$.

Estudos sobre a geração de modelos da morfologia para PA6/SAN compatibilizados com polímeros funcionalizados tem mostrado que alguns dos polímeros compatibilizantes deixam a fase SAN e residem como estruturas micelar na fase poliamida. É razoável prever que este fenômeno se estende para as blendas PA6/ABS compatibilizadas com o SMA. Durante o processamento, alguns dos grupos anidrido do SMA são convertidos em unidades imida através da reação com a PA6 (Figura 1); em última instância, isto poderia mudar a estrutura molecular do SMA de modo que ela deixaria de ser miscível com o SAN do ABS. Como as moléculas de SMA modificadas perdem afinidade com o SAN, os polímeros enxertados podem formar micelas na fase poliamida. A existência de agregados finamente dispersos do SMA frágil na fase poliamida, aliada ao grande tamanho de domínios da fase dispersa ABS, podem ser as causas da perda de tenacidade para estas blendas ${ }^{[1]}$.

\section{Propriedades mecânicas}

A Figura 6 e Tabela 1 mostram as propriedades de rigidez e tenacidade para os polímeros puros, para a blenda PA6/ABS e para as blendas preparadas através das três sequências de mistura. A presença de $40 \%$ de $\mathrm{ABS}$ na blenda não alterou muito a rigidez da PA6 uma vez que o resultado obtido para o módulo desta foi bem próximo ao encontrado para a matriz PA6. Com relação às sequências de mistura, observa-se que os valores de módulo encontrados foram bem próximos e menores que o apresentado pela matriz.

Os valores de resistência ao impacto apresentados pelas diferentes sequências, Figura 6b, ao contrário dos resultados de módulo de elasticidade, foram bastante diferentes. A mistura simultânea de todos os componentes (PA6/ABS/SMA) foi a mais eficaz no processo de tenacificação, onde foi encontrado um aumento de $57 \%$ em relação à matriz. Já o sistema (ABS/SMA) + PA6 apresentou uma redução na tenacidade em relação à matriz e à blenda PA6/ABS sem o compatibilizante. $\mathrm{O}$ aspecto de fratura poder ser considerado frágil em função dos valores obtidos. O resultado apresentado pela sequência (PA6/SMA) + ABS foi bem próximo ao apresentado pela PA6, logo essa sequência de mistura não se mostrou eficaz em melhorar a tenacidade da matriz. Essas diferenças de comportamento apresentadas pelos materiais preparados por essas sequências de mistura provavelmente estão relacionadas com a morfologia gerada durante o processamento destas blendas. As imagens de MET mostram que a blenda PA6/ABS/SMA apresentou uma redução do tamanho dos domínios da fase dispersa quando comparada com os domínios da blenda sem compatibilizante PA6/ABS. Por outro lado, os domínios observados da fase ABS na blenda (ABS/SMA) + PA, apresentaram-se mais grosseiros e mais alongados em relação àqueles da blenda PA6/ABS. Além disso, a presença de uma outra fase na matriz PA6 pode contribuir para a redução da tenacidade observada por este sistema, conforme já discutido anteriormente.

A morfologia gerada pela sequência (PA6/SMA) + ABS, aparentemente foi a mais estável. Foi observada uma redução bastante acentuada no tamanho dos domínios da fase dispersa para esta blenda. Esperava-se então, que este sistema apresentasse um maior valor de tenacidade. Um provável fator que pode estar relacionado com o comportamento apresentado por esta blenda, é o nível de reação de enxertia que ocorre na interface PA6/ABS devido a formação in situ do copolímero PA6-g-MA. A maneira segundo a qual esta mistura foi realizada, conforme já mencionado, pode favorecer a um excesso de copolímero enxertado, o qual perde a sua efetividade como um compatibilizante.

\section{Temperatura de Deflexão Térmica (HDT)}

A incorporação de ABS como modificadores de impacto para polímeros de engenharia, em relação a outros modificadores de impacto elastoméricos, tem como vantagem a melhora, ou pelo menos, o não abaixamento da temperatura de distorção térmica $(\text { HDT })^{[17]}$.

Para avaliar esse comportamento foram ensaiados os polímeros puros, as blendas binárias e ternárias. Os resultados obtidos estão apresentados na Figura 7. A HDT da PA6 pura ficou na faixa de $55^{\circ} \mathrm{C}$. Foi observado um aumento bastante significativo na HDT das blendas compatibilizadas, como nas blendas sem compatibilizante. A incorporação do ABS que apresenta mais alto valor de HDT em relação à matriz contribuiu para o correspondente aumento desta propriedade. Os valores observados para estas blendas são muito
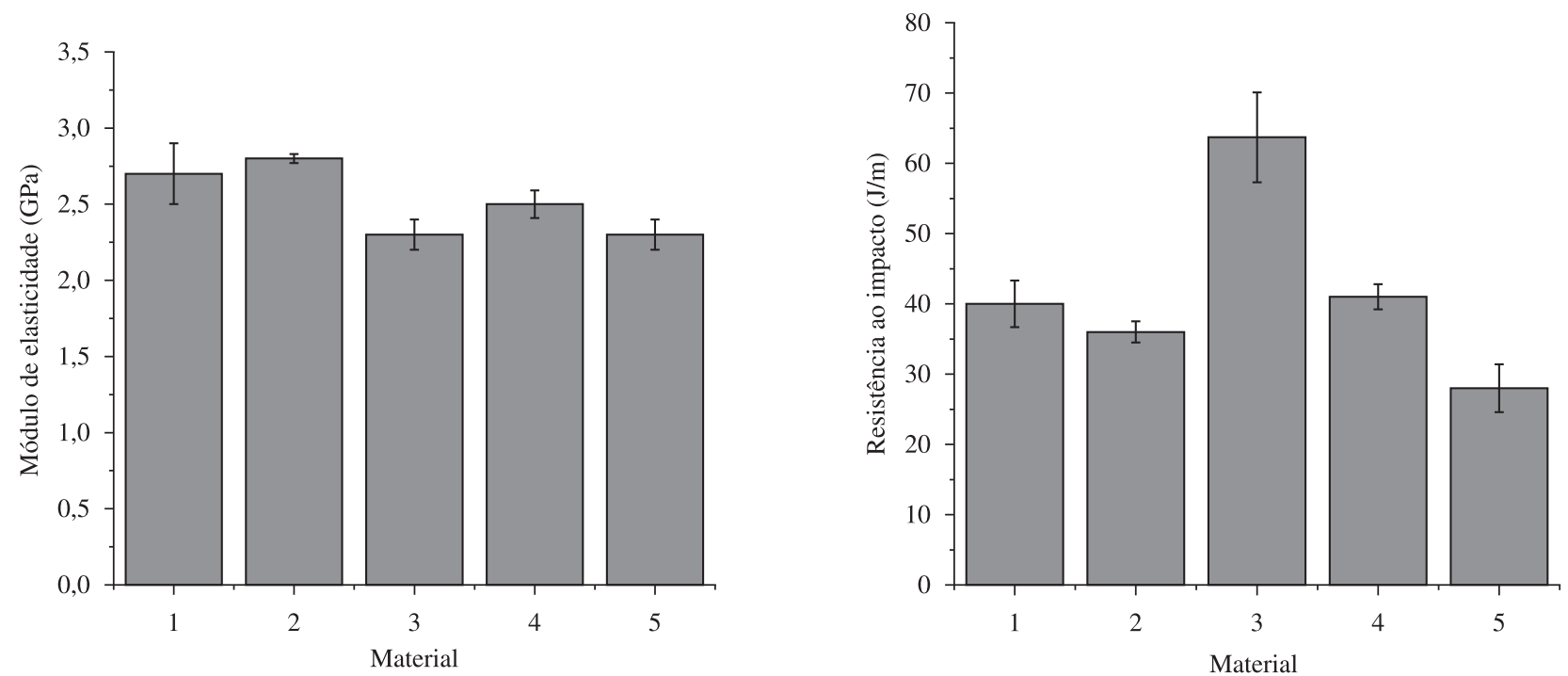

Figura 6. Módulo de elasticidade e resistência ao impacto dos polímeros puros e das blendas PA6/ABS, compatibilizadas e não compatibilizadas: 1) PA6; 2) PA6/ABS; 3) PA6/ABS/SMA; 4) (PA6/SMA) + ABS; e 5) (ABS/SMA) + PA6. 


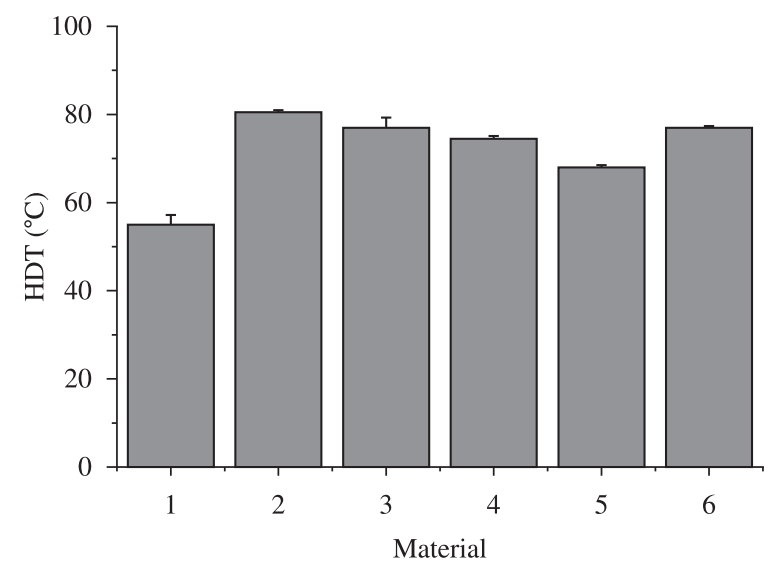

Figura 7. HDT da PA6 pura, da blenda PA6/ABS e das blendas compatibilizadas com o copolímero SMA: 1) PA6; 2) ABS; 3) PA6/ABS; 4) PA6/ABS/SMA; 5) (PA6/SMA) + ABS; e 6) (ABS/SMA) + PA6.

interessantes do ponto de vista tecnológico, pois eles são superiores aos da PA6. Como já foi observado, o módulo apresentado por estas blendas foi bem próximo ao encontrado pela matriz PA6, ou seja, mantiveram a sua rigidez.

\section{Conclusões}

Neste trabalho, blendas de PA6/ABS compatibilizadas com o copolímero SMA foram estudadas. A blenda sem compatibilizante PA6/ABS pode ser considerada frágil e incompatível, devido à baixa interação molecular entre os componentes, bem como à má dispersão dos domínios de ABS. As morfologias e propriedades mecânicas das blendas compatibilizadas com o copolímero SMA são bastante diferentes e dependem da sequência de mistura. Pode-se concluir que a mistura simultânea de todos os componentes (PA6/ABS/SMA) foi a mais eficaz no processo de tenacificação. Evidências de reações químicas entre a PA6 e o copolímero SMA foram observadas através de reometria torque. Um aumento no valor do torque indica a ocorrência de reações químicas entre o MA do SMA e os grupos finais de cadeia a PA6 e, consequentemente, a formação de moléculas de copolímero PA6-g-MA.

\section{Agradecimentos}

Os autores agradecem ao $\mathrm{CNPq}$ pelo apoio financeiro e as petroquímicas BASF e SABIC pela doação dos polímeros.

\section{Referências Bibliográficas}

1. Chiang, C. R. \& Chang, F. C. - Polymer, 38, p.4807 (1997).

2. Bassani, A.; Pessan, L. A. \& Hage Jr, E. - Polímeros, 12, p.102 (2002).

3. Araújo, E. M.; Hage Jr, E. \& Carvalho, A. J. F. - Polímeros, 14, p.22 (2004).

4. Li, Y. \& Shimizu, H. - Macromol. Rapid. Comm., 26, p.710 (2005).

5. Kudva, R. A.; Keskkula, H. \& Paul, D. R. - Polymer, 41, p.225 (2000).

6. Kudva, R. A.; Keskkula, H. \& Paul, D. R. - Polymer, 41, p.239 (2000).

7. Jafari, S. H.; Potschke, P.; Stephan, M.; Warth, H. \& Alberts, H. Polymer, 43, p.6985 (2002).

8. Cho, K.; Seo, K. H. \& Ahn, T. O. - J. Appl. Polym. Sci., 68, p.1925 (1998).

9. Kim, B. K.; Lee, Y. M. \& Jeong, H. M. - Polymer, 34, p.2075 (1993).

10. Majumdar, B.; Keskkula, H. \& Paul, D. R. - Polymer, 35, p.3164 (1994).

11. Li, Q.; Tian, M.; Kim, D.; Zhang, L. - J. Appl. Polym. Sci., 85, p.2652 (2002).

12. Kim, B. K.; Lee, Y. M. \& Jeong, H. M. - Polymer, 34, p.2075 (1993).

13. Choi, J. H.; Kim, H.; Han, D. H.; Lim, J.C. - J. Appl. Polym Sci, 101, p.1 (2006).

14. Hale, W.; Keskkula, H.; Paul, D. R. - Polymer, 40, p.3665 (1999).

15. Dedecker, K.; Groeninckx, G. - Macromolecules, 32, p.2472 (1999).

16. Araújo, E. M; Hage JR., E.; Carvalho, A. J. F. - Polímeros: Ciência e Tecnologia, 13 p. 205 (2003).

17. Mantovani, G. L. - “Compatibilização por extrusão reativa de blendas poliméricas PBT/ABS e estudo da estabilidade da morfologia de fases", Tese de Doutorado em Ciência e Engenharia de Materiais, Programa de Pós-Graduação em Ciência e Engenharia de Materiais (PPG-CEM/UFSCar), São Carlos, Brasil (2002).

Enviado: $18 / 03 / 10$

Aceito: $22 / 07 / 10$

DOI: $10.1590 /$ S0104-14282011005000010 